\title{
Preventive Control for Transient Security with Generation Rescheduling Based on Rotor Trajectory Index
}

\begin{abstract}
Kusum Verma ${ }^{\dagger}$ and K. R. Niazi*
Abstract - The increasing need to improve transient security assessment of existing or forecasted operating conditions of networks by power system operators is major concern of the power system security monitoring problem at the Energy Management Systems. This paper proposes a preventive control of transient stability with generation rescheduling based on rotor trajectory index obtained using time domain simulations. This index may help power engineers in making operational decision and to obtain a generation configuration with better transient security dispatch. The effectiveness of the proposed methodology is demonstrated on IEEE 39-bus New England system for a three phase fault at different loading conditions with single and multiple line outage cases.
\end{abstract}

Keywords: Generation rescheduling, Preventive control, Rotor trajectory index, Transient security

\section{Introduction}

Security control is one of the prime concerns for planning and operation of modern power systems. The increase in size and complexity of modern interconnected power systems and the present trend towards deregulation has forced the electric utilities to operate their systems under stressed operating conditions closer to their security limits [1]. With this growing stress on present day power systems, they are increasingly facing the threat of transient stability problems. Transient stability or large disturbance rotor angle stability is concerned with the ability of the power system to maintain synchronism when subjected to a severe disturbance, such as a short circuit on a transmission line. Instability that may result occurs in the form of increasing angular swings of some generators leading to their loss of synchronism with other generators ([2, 3]). When the transient security level falls below an acceptable level, preventive control actions can restore the system back to secure state of operation [4].

Preventive control aims at modifying the operating conditions of a power system so as to make it able to withstand severe contingencies that would drive it to instability, whenever they occur. The types of preventive control actions include: generation rescheduling; network switching; reactive compensation; and load curtailment. Preventive control is summoned up when the power system is still in normal status [5]. In the past many utilities have relied on preventive control in order to maintain system security at an acceptable level. This is due to the fact that with preventive control time is available for decision

$\dagger$ Corresponding Author: Department of Electrical Engineering, Malaviya National Institute of Technology, Jaipur, Rajasthan, India. (kusum_dangi@yahoo.com)

* Department of Electrical Engineering, Malaviya National Institute of Technology, Jaipur, Rajasthan, India. (krn152001@yahoo.co.in)

Received: June 28, 2014; Accepted: September 25, 2014 making. Also, for the uncertain disturbances, the state of system is known in this control; therefore, it becomes easier to study dynamic behavior of the system. The core of power system operation is to maintain a balance between supply and demand of electric power. Since reactive power can be locally compensated, active power balance is the most important issue for power system operation.

Preventive control is mainly dominated by generation rescheduling as reported in the literature survey. Various generator rescheduling techniques [6-23] have been proposed for transient security control. In [6], the transient energy function (TEF) method is used to check the dynamic security of the system and the coherent behavior of generators is used to find a new generation configuration. In [7], a coherency based sensitivity method is proposed for generation rescheduling. In [8], a coherency based generator rescheduling for preventive control of transient stability in power systems is proposed. In [9], dynamic security assessment and generation rescheduling method utilizing genetic algorithms (GAs) integrated with probabilistic neural networks (PNNs) and adaptive neurofuzzy inference systems (ANFISs) is proposed for the preventive control power systems against transient instabilities. A generation rescheduling approach for preventive control of power system is presented in [10] to reallocate power generation for multiple unstable contingencies based on graph theory. In [11], proposed a transient stability preventive control design that takes series compensation into account. A heuristic stability performance index (PI) was proposed to evaluate the gradient of the PI and determine the preventive control accordingly [12]. In [13], rescheduling of power systems constrained with transient stability limits in restructured power systems based on TEF is proposed. An optimal generation rescheduling with trajectory sensitivity-based 
transient stability constraints is presented in [14]. Generation rescheduling based upon the optimal result of multi-contingency transient stability constrained optimal power flow is suggested as preventive control of power systems in [15]. A real time preventive action for transient stability enhancement with a hybrid neural networkoptimization approach using derivatives of transient energy margin value is proposed in [16]. A technique using trajectory sensitivities to provide a preventive rescheduling scheme in dynamic security constrained power systems taking into account the economic aspect is proposed in [17]. In [18], trajectory sensitivities are used for multi-contingency preventive control. Iterative solution methodology and computer package for generation rescheduling is suggested in [19]. The proposed approach in [20] considers generator shedding as the most effective discrete supplementary control for improving the dynamic performance of faulted power systems and preventing instabilities considering the sensitivity of the TEF with respect to changes in the amount of dropped generation which is used during the training phase of ANNs to assess the critical amount of generator shedding required to prevent the loss of synchronism. Decision tree (DT)-based preventive and corrective control methods are proposed in [21] to enhance the dynamic security of power systems against the credible contingencies causing transient instabilities. A radial basis function (RBF) neural network to assess the dynamic security status of the power system and to estimate the effect of a corrective control action applied in the event of a disturbance is proposed in [22] with optimal control action using particle swarm optimization (PSO). In [23], an optimal power flow model with transient stability constraints is proposed for both dispatching and re-dispatching.

The individual machine synchronism state for a given operating condition needs to be monitored to decide the participating generators for rescheduling to ensure security of power systems under all operating conditions. There is need for thorough investigation to obtain secure precontingency generating schedules so that system remains transiently secure in the event of large disturbances. For any approach using the energy margin and sensitivity analysis, computation time is a concern and a somewhat different approach is required for determining the proper preventive control action for transient security improvement. Therefore, there is an urgent need to improve and develop more robust and efficient preventive control method for transient security control. The objective of this paper is to analyze the role of preventive control from a transient security perspective and to develop alternative approach for active power rescheduling of generators to improve power system preventive control plans. The proposed approach is based on transient security index for preventive control of power system with multiple unstable contingencies. The method also determines the individual machine synchronism state for a given operating condition.
The method have been tested on IEEE 39-bus, 10generator system and the transiently insecure cases for certain contingencies are made secure by rescheduling the generation according to the proposed method. Application results show that proposed method is able to provide nearoptimal control solutions meeting steady-state and transient stability constraints.

\section{Power System Swing Equation}

The transient security assessment (TSA) requires dynamic simulation of power system to determine whether synchronism is preserved following a disturbance. This is judged by observing the variations of rotor angles following a disturbance over a period of time. If the rotor angle $\delta$ of a machine or a group of machines continues to increase without bound with respect to rest of the system, the system is called unstable. On the other hand if all rotor angles remain bounded within limit around their post disturbance steady state values, then the system is called stable system. The dynamic behavior of an $n$-generator power system can be described using the following swing equations $([2,8])$ :

$$
\begin{gathered}
\frac{d \delta_{i}}{d t}=\Delta \omega_{i} \\
\frac{d \Delta \omega_{i}}{d t}=\frac{1}{M_{i}}\left(P_{m i}-P_{e i}-D_{i} \Delta \omega_{i}\right) \\
P_{e i}=G_{i i} E_{i}^{2}+\sum_{j=1, j \neq i}^{n} E_{i} E_{j}\left\{G_{i i} \cos \left(\delta_{i}-\delta_{j}\right)\right. \\
\left.+B_{i j} \sin \left(\delta_{i}-\delta_{j}\right)\right\}
\end{gathered}
$$

where, for $i^{\text {th }}$ machines $\delta_{i}$ is rotor angle, $\Delta \omega_{i}$ is rotor speed deviation, $P_{m i}$ is mechanical power input, $P_{e i}$ is electrical power output, $M_{i}$ is moment of inertia, $D_{i}$ is damping coefficient, $\delta_{j}$ is rotor angle of $j{ }^{\text {th }}$ machine, $G_{i j}+j B_{i j}$ is transfer admittance between the $i^{\text {th }}$ and $j^{\text {th }}$ machines. In describing the transient behavior of the system, it is convenient to use inertial center as a reference frame. The generators' angles with respect to centre of inertia (COI) [18] are used to indicate whether or not the system is stable. For an $N_{G}$-generator system with rotor angles $\delta_{i}$ and inertia constant $M_{i}$, the position of the inertial center $\delta_{C O I}$ is defined as

$$
\delta_{C O I}=\frac{\sum_{i=1}^{N G} \delta_{i} M_{i}}{\sum_{i=1}^{N G} M_{i}}
$$

In this paper, swing equation is solved by numerical integration techniques such as Runge-Kutta method. By observing the swing curves, the relative rotor angle of each generator with respect to COI are obtained to assess the transient security status. The criterion for transient instability is given as

$$
\Delta \delta_{i, \mathrm{COI}}=\left|\delta_{i}-\delta_{\mathrm{COI}}\right| \leq \delta_{\max } \text { for } \mathrm{i}=1 \text { to } \mathrm{N}_{\mathrm{G}}
$$


Here, $\delta_{i}$ is the rotor angle of $i^{\text {th }}$ generator and $\delta_{\max }$ is maximum allowable relative rotor angle difference for secure operation. If there is no relative rotor angles violations of any generator with respect to COI, $\Delta \delta_{i, C O I}$ $\left(\leq \delta_{\max }\right)$ after fault clearing, the system is said to be secure $(1)$, else insecure (0). For this study $\delta_{\max }$ is taken as $120^{\circ}$ $([22,23])$.

\subsection{Rotor Trajectory Index (RTI)}

The rotor trajectory index $(R T I)$ can be used to measure the synchronism state of a generating unit and hence stability of the system for given severity of the contingency. In this paper, an angle based stability index $R T I$ ([6-8]), is used in order to assess the severity of a contingency and the system's trajectory following a disturbance, defined as

$$
R T I=1-\frac{360^{\circ}-\left(\Delta \delta_{i, C O I}\right)_{\max }}{360^{\circ}+\left(\Delta \delta_{i, C O I}\right)_{\max }}
$$

where $\left(\Delta \delta_{i, C O I}\right)_{\max }$ is the maximum relative rotor angle difference of any generator with respect to COI in the post-fault response, obtained using (5). Here, $R T I>\varepsilon$ and $R T I \leq \varepsilon$ correspond to unstable and stable conditions, respectively and the generating machines can be grouped into critical machines, $C M s$ and non-critical machines, $N M s$ respectively. For a given post-fault condition, if any generating unit have $R T I>\varepsilon$, it indicates that this machine will lose synchronism with rest of the system making it insecure. The severity of the machines in order of their losing synchronism can be obtained by their RTI value that gives their ranking. Thus severity of machines can be analyzed on basis of the value of this index and accordingly significant units can be determined to apply preventive control for a given contingent case.

\section{Proposed Methodology for Generation Rescheduling}

Generation rescheduling has long been recognized as an efficient and cost effective means to alleviate system transient insecurity [14]. Generation rescheduling aims at shifting generation from selected machines. The proposed method utilizes the information of the rotor trajectory index values to determine the significant units to be considered for rescheduling. The pre-contingent power flow on the line is also distributed among the candidate machines for a given operating condition so as to maintain the power balance between supply and demand. The slack generator is not included in the rescheduling process as the active power generation of this generator is not controllable. This generator is used to provide system losses and to balance the active and reactive power in the system.

\subsection{Selection of generating units for rescheduling}

After the determination of critical contingencies, the generating units to be rescheduled are determined by their generating capabilities and their effects on restoring the security for the specified contingencies. In order to assess the severity of a contingency, the system's trajectory following a disturbance and hence the severity of machines in terms of their synchronism can be analyzed on basis of the value of this index. The machines having $R T I>\varepsilon$ are the "critical machines" $(C M S)$ and the machines having $R T I \leq \varepsilon$ are the "non-critical machines" $(N M S)$. Accordingly significant units can be determined for a given insecure contingent case and preventive control may be applied to these units to restore system security. The flowchart for proposed preventive control method based on rotor trajectory index, RTI is shown in Fig.1.

The procedure for proposed preventive control based on $R T I$ values is summarized as follows:

1) For the insecure contingency, all the machines are divided into groups based on their RTI as the CMs and NMs.

2) All the critical machines having $R T I>\varepsilon$, form a group known as Generation Decrease $(G D)$ group. All the non-critical machines having $R T I \leq \varepsilon$, form a group known as Generation Increase ( $G I)$ group.

3) The non-critical machine having lowest value of $R T I$ is first considered to take up the increase in active power generation. The machines of GI group are the candidate generating units that are available and controllable for re-dispatch.

4) Synchronous generators running in parallel and delivering active power to the load depends on the rotor angle of the generators. This shows that any change in relative rotor angles will affect the active power output of the generating units. Rotor angle stability must be maintained for inter-connected synchronous machines running in the power system to remain in the state of synchronism. In this paper an index is developed to find the amount of generation decrease / increase for the generating units to regain synchronism in the event of insecure contingencies. Based on RTI values, a Generation Shift Factor, GSF is proposed for all insecure generators / critical machines having $R T I>\varepsilon$. For each $i^{\text {th }}$ critical machine, $G S F_{i}$ is defined as

$$
G S F_{i}=\frac{\delta R T I_{i}}{\delta P_{G i}}=\frac{\Delta R T I_{i}}{\Delta P_{G i}}=\left|\frac{R T I_{i}^{\text {post }}-R T I_{i}^{\text {pre }}}{P_{G i}^{\text {post }}-P_{G i}^{\text {pre }}}\right|
$$

where, $i \in G D$

where, $R T I_{i}{ }^{\text {post }}=$ post-contingent value of rotor trajectory index for $i^{\text {th }}$ critical machine

$R T I_{i}{ }^{\text {pre }}=$ pre-contingent value of rotor trajectory index for $i^{\text {th }}$ critical machine 


$$
\begin{aligned}
P_{G i}{ }^{\text {post }}= & \text { post-contingent real power output of } i^{t h} \text { critical } \\
& \text { machine } \\
P_{G i}{ }^{\text {pre }}= & \text { pre-contingent real power output of } i^{\text {th }} \text { critical } \\
& \text { machine }
\end{aligned}
$$

The amount of real power shift, $\Delta P_{G i}$ shift from these critical machines of $G D$ group is then determined using the following equation:

$$
\Delta P_{G i}^{\text {shift }}=G S F_{i} \times P_{G i}^{\text {pre }}
$$

5) The real power generation of the most stressed $C M s$ is decreased as per Eq. (8) and their new schedules, $P_{G i}{ }^{\text {new }}$ are calculated using

$$
\mathrm{P}_{\mathrm{Gi}}^{\text {new }}=\mathrm{P}_{\mathrm{Gi}}{ }^{\text {pre }}-\Delta \mathrm{P}_{\mathrm{Gi}} \text { shift }
$$

For each case, the decrease of generation in $C M s$ is done first for the most severe unit with rank 1 as per RTI.

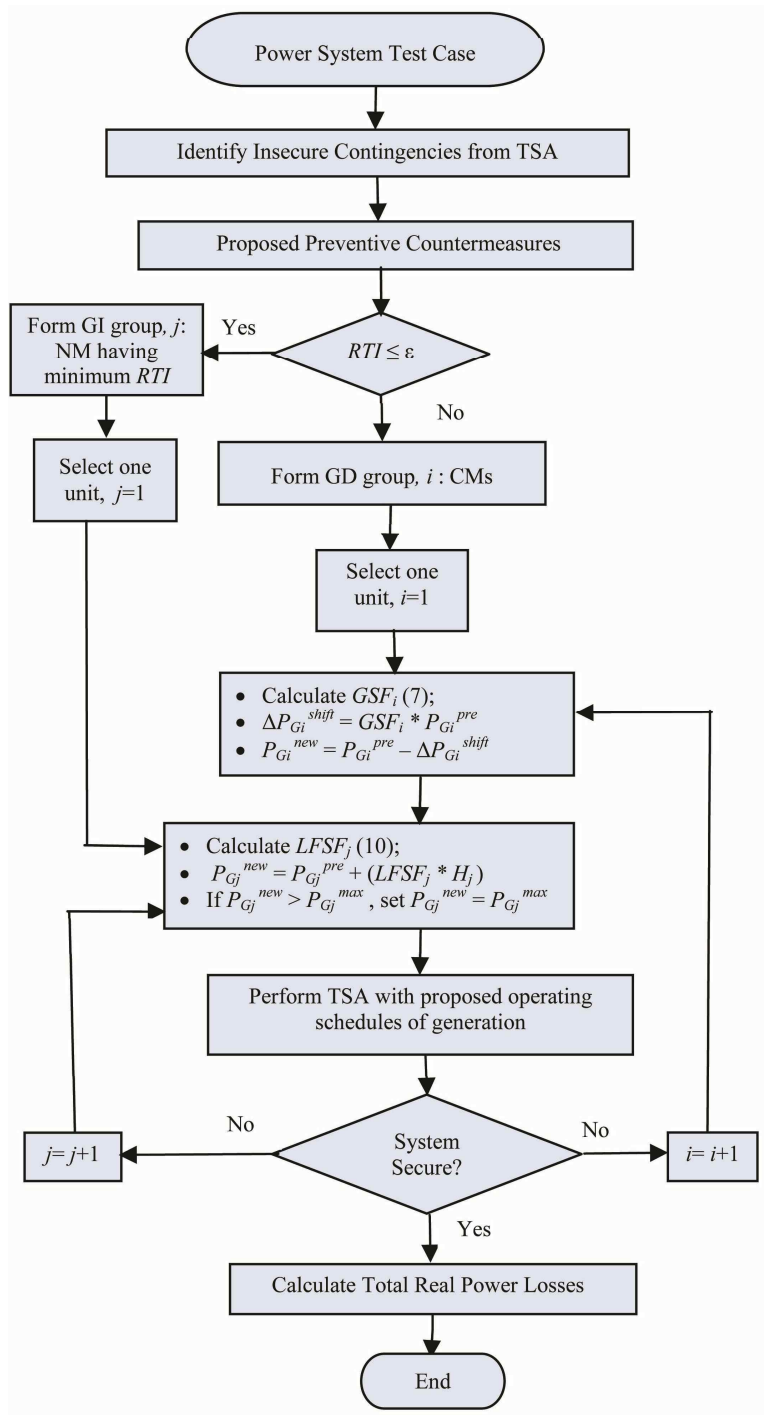

Fig. 1. Flowchart of proposed preventive control method based on rotor trajectory index
6) Pre-fault transmission line power flows may be modified to remove contingency problems. In an operational context this requires working with the existing electric grid, typically involving re-dispatch of generation so that power balance may be maintained. Therefore, the pre-contingent real power flow on the outaged line may be carried by the non-critical machines.

7) Real power rescheduling of each $j^{\text {th }}$ candidate noncritical machine is done by increasing their generation to adjust the decrease done for $C M s$ and to carry additional pre-contingent real power flow on the outaged line. This is determined according to the proposed Line Flow Shift Factor, $L F S F_{j}$, defined as

$$
\begin{array}{cc}
L F S F_{j}=\frac{\Sigma \Delta P_{G i}^{s h i f t}+\sum P_{G j}^{\text {pre }}+\sum P_{L O}{ }^{\text {pre }}}{\left.\sum{\left(P_{G j}\right.}^{\text {pre }} * H_{j}\right)} \\
\text { where, } \\
j \in G I
\end{array}
$$

where, $\Delta P_{G i}{ }^{\text {shift }}=$ Real power decrease of each $i^{\text {th }}$ critical machine

$P_{G j}{ }^{p r e}=$ Pre-contingent real power output of $j^{\text {th }}$ noncritical machine

$P_{L O}$ pre $=$ Pre-contingent real power flow on the outaged line

$H_{j}=$ Inertia constant of $j^{\text {th }}$ non-critical machine

8) In order to make the system secure, the real power generation of the NMs is increased as per Eq. (10) and their new schedules, $P_{G j}{ }^{\text {new }}$ are calculated using

$$
P_{G j}{ }^{\text {new }}=P_{G j}^{\text {pre }}+\left(L F S F_{j} \times H_{j}\right)
$$

The amount of generation increment is distributed in each unit of GI group in proportion to their machine inertias.

9) For obtaining secure operation, the active power generation constraint for candidate $N M s$ must be satisfied as: $P_{G j}{ }^{\text {new }}<=P_{G j}{ }^{\max }$, where, $P_{G j}{ }_{\max }$ is the maximum limit of active power generation of $j^{\text {th }}$ noncritical generating machine.

10) To obtain a transiently secure post-contingent scenario, the pre-contingent operating point is obtained by rescheduling the generating units according to these new active power schedules.

11) If the system is found to secure after applying the proposed reschedule, the rescheduling is complete; otherwise, the decrease of generation in CMs is done for other units in decreasing order of their ranks as per $R T I$. For generation increase, one more non-critical machine with next lowest value of $R T I$ is included in the rescheduling process.

12) The process is continued till a secure state of the system is obtained.

13) After obtaining transient secure state with the proposed rescheduling, the total real power losses for the system are calculated and the process terminates. 


\section{Simulation and Results}

The proposed method is tested on the IEEE-39 bus 10 -generator New England system. The system has 10 generators, 12 transformers, 46 transmission lines shown in Fig. 2. Bus no. 39 is taken as slack bus [24]. It is $345 \mathrm{kV}$ dynamic test system. The bus voltage magnitude limits are $V^{\min }=0.94$ p.u. and $V^{\max }=1.06$ p.u. All the simulations are carried out using MATLAB 7.7 [25], PST v3[26] with Intel Core i5, $2.50 \mathrm{GHz}, 6 \mathrm{~GB}$ RAM.

Three sample cases are illustrated in this section for IEEE 39-bus, 10-generator system for different contingent scenarios to demonstrate the effectiveness of the proposed methods. The three phase short circuit to ground fault is applied at $0.01 \mathrm{~s}$ and is cleared by opening the line 26-28. The fault duration is $80 \mathrm{~ms}$ and time domain simulation (TDS) is performed for $1 \mathrm{sec}$. with frequency $60 \mathrm{~Hz}$ to find whether the system is secure or insecure after a fault is

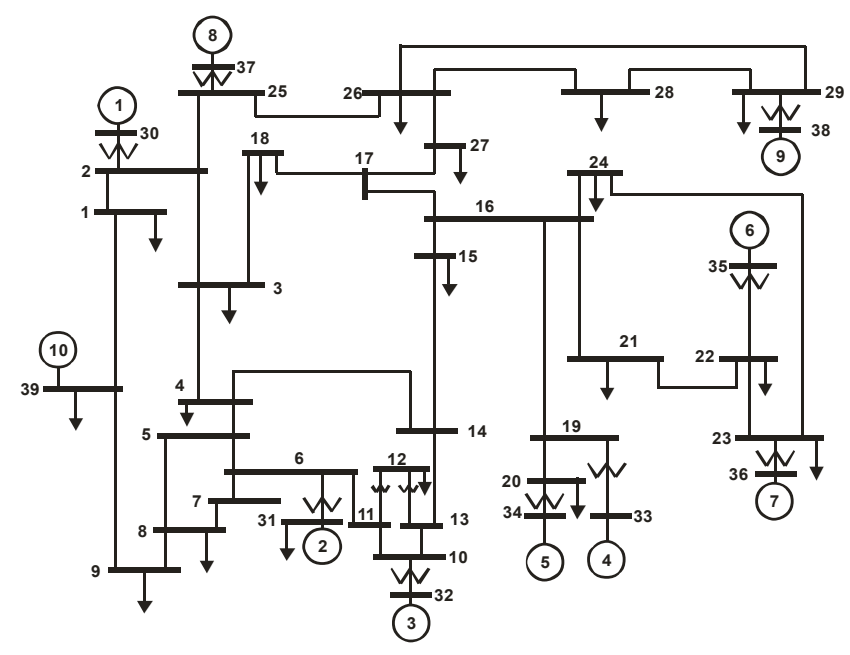

Fig. 2. Single line diagram of IEEE 39-bus, 10-generator new england system cleared.

For each case, a new pre-contingent operating point is determined, which is based on the results of proposed rescheduling. The TDS is carried out with the proposed generation schedules to establish post-contingent transient secure system and the performance analysis is carried out by determining the security status and total real power losses of the system. The proposed method reschedules the active generation of the units using Eqs. (7) - (11). The generation is decreased for $G D$ units according to $G S F_{i}(7)$ and new active power schedules for these units are obtained using (8) and (9). The generation is increased for $G I$ units according to $L F S F_{j}(10)$ and new active power schedules for these units are obtained using (11). The obtained results of the proposed rescheduling for each case are verified by observing the transient stability/instability scenario, before and after rescheduling, for each case on the basis of the relative rotor angle swings with respect to COI obtained by the numerical routines for $1 \mathrm{sec}$.

\section{Case I: N-1 Topology Variation; 97.5\% of Base Case}

This case study is shown for $97.5 \%$ of base case with random load variation and $\mathrm{N}-1$ contingency selection. Single line outage of line $2-25$ is considered with threephase fault at bus-26. As shown in Table 1, for line outage of line 2-25, TDS results show that generator no. 2, 5, 9 goes out-of-step with respect to $\mathrm{COI}$ and the system becomes transiently insecure. For each generating unit, $R T I>\varepsilon$ indicates that the machine lose synchronism making the system insecure. As seen from the post-contingent value of $R T I^{\text {post }}$, the generator no. 5, 2 and 9 loses synchronism, in the order of their severity ranking with rest of the system. Therefore, these generating units make the system transiently insecure. To bring back system to secure state, generation rescheduling is required. The generation may be shifted from these insecure $G D$ units $(C M s)$ to the candidate secure $G I$ units $(N M s)$.

Table 1. Results obtained from time domain simulations for IEEE 39-bus system at $97.5 \%$ of base case with N-1 topology variation (insecure case)

\begin{tabular}{|c|c|c|c|c|c|c|c|c|}
\hline Generator Number & Bus Number & $P_{G}^{\max }$ (p.u.) & $P_{G}^{\text {pre }}$ (p.u.) & $P_{G}^{\text {post }}$ (p.u.) & $R T I^{\text {pre }}$ & $R T I^{\text {post }}$ & Severity Rank & $\mathrm{H}(\mathrm{sec})$ \\
\hline 1 & 30 & 10.4 & 6.563 & 6.8977 & 0.160 & 0.362 & - & 4.2 \\
\hline 2 & 31 & 6.46 & 6.460 & 7.0256 & 0.321 & 0.505 & 2 & 3.03 \\
\hline 3 & 32 & 7.25 & 6.556 & 7.1892 & 0.252 & 0.449 & - & 3.58 \\
\hline 4 & 33 & 6.52 & 6.393 & 7.1160 & 0.299 & 0.493 & - & 2.86 \\
\hline 5 & 34 & 5.08 & 5.080 & 5.7537 & 0.331 & 0.533 & 1 & 2.6 \\
\hline 6 & 35 & 6.87 & 6.452 & 6.9164 & 0.259 & 0.459 & - & 3.48 \\
\hline 7 & 36 & 5.80 & 5.800 & 6.4612 & 0.301 & 0.482 & - & 2.64 \\
\hline 8 & 37 & 5.64 & 5.640 & 5.6698 & 0.311 & 0.473 & - & 2.43 \\
\hline 9 & 38 & 8.65 & 6.384 & 5.9789 & 0.302 & 0.501 & 3 & 3.45 \\
\hline
\end{tabular}

Table 2. Active power rescheduling results for IEEE 39-bus system for Case-I obtained from proposed method

\begin{tabular}{c|c|c|c|c|c|c}
\hline GD unit $(i)$ & $\Delta P_{G i}{ }^{\text {sijf }}$ (p.u.) & $P_{G i}^{\text {new }}$ (p.u.) & Line Outage & $P_{L O}{ }^{\text {pre }}$ (p.u. $)$ & GI unit $(j)$ & $P_{G j}{ }^{\text {ew }}($ p.u. $)$ \\
\hline 2 & 2.1016 & 4.3584 & $2-25$ & 1.6657 & 1 & 8.8469 \\
\hline 5 & 1.5232 & 3.5568 & & & & \\
\hline 9 & 3.1361 & 3.2479 & & & & \\
\hline
\end{tabular}


Table 2 show the proposed rescheduling of the active power generations among $C M S$ and $N M S$ to bring the current insecure operating state to a secure state. It is found that the GI group consists of only one unit, which is generator no.1 having lowest value of RTI post. The generation decrease is required for all three critical machines while generation increase for only one non-critical generating unit is sufficient to bring the system back to secure state. As seen from Fig. 3, relative rotor angle of generator no. 2, 5 and 9 increases beyond the threshold value, $\varepsilon$ and goes out of step before rescheduling. These machines lose synchronism with rest of the generators making system transiently insecure. As shown in Fig. 4, after proposed rescheduling, the relative rotor angle of all the generating units is below $\varepsilon$ and the system becomes secure.

\section{Case II: N-2 Topology Variation; Base Case}

This case study is shown for base case with random load variation and N-2 contingency selection. Single line outage of line 2-3 and line 14-15 is considered with three-phase fault at bus-26. Table 3 shows the results obtained from

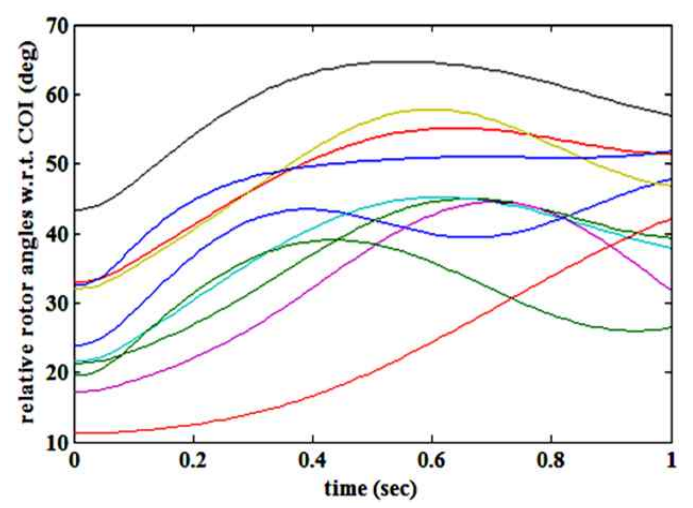

Fig. 3. Relative rotor angle trajectories of all generators of IEEE 39-bus system before rescheduling for Case I (insecure case) time domain simulations for this case. As seen from the post-contingent value of $R T I^{\text {post }}$, the generator no. 5 loses synchronism with rest of the system.

Table 4 shows the proposed rescheduling of the active power generations among $C M S$ and $N M S$ to bring the current insecure operating state to a secure state. It is found that the GI group consists of only one unit, which is generator no. 1 having lowest value of RTI post. It is found that the GI group consists of only one unit, which is generator no.1 having lowest value of RTI post. The generation decrease is required for one critical machine while generation increase is required for one non-critical generating unit to bring the system back to secure state.

As seen from Fig. 5, relative rotor angle of generator no. 5 increases beyond the threshold value, $\varepsilon$ and goes out of step before rescheduling. This machine loses synchronism with rest of the generators making system transiently insecure. To make the system secure for this case, the proposed rescheduling is applied to the generating units. As shown in Fig. 6, after proposed rescheduling, the relative rotor angle of all the generating units is below $\varepsilon$ and the system becomes secure.

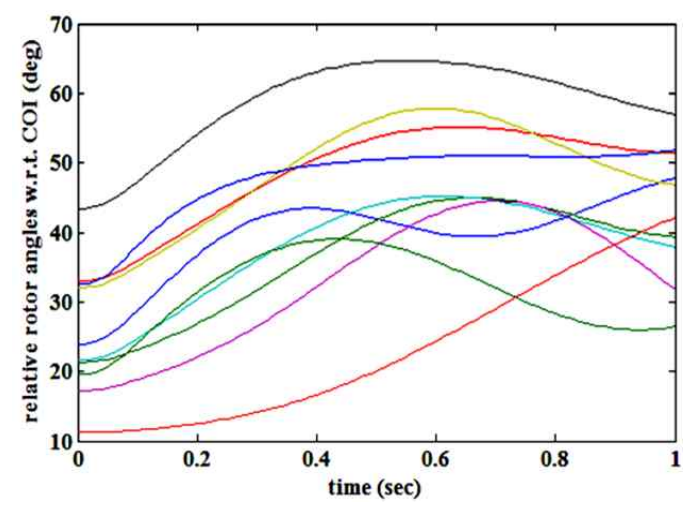

Fig. 4. Relative rotor angle trajectories of all generators of IEEE 39-bus system after proposed rescheduling for Case I (secure case)

Table 3. Results obtained from time domain simulations for IEEE 39-bus system at base case with N-2 topology variation (insecure case)

\begin{tabular}{|c|c|c|c|c|c|c|c|c|}
\hline Generator Number & Bus Number & $P_{G}^{\max }$ (p.u.) & $P_{G}{ }^{\text {pre }}$ (p.u.) & $P_{G}{ }^{\text {post }}$ (p.u.) & $R T I^{\text {pre }}$ & $R T I^{\text {post }}$ & Severity Rank & $\mathrm{H}(\mathrm{sec})$ \\
\hline 1 & 30 & 10.4 & 6.819 & 6.4306 & 0.164 & 0.350 & - & 4.2 \\
\hline 2 & 31 & 6.46 & 6.460 & 6.9024 & 0.318 & 0.437 & - & 3.03 \\
\hline 3 & 32 & 7.25 & 6.814 & 7.3239 & 0.255 & 0.382 & - & 3.58 \\
\hline 4 & 33 & 6.52 & 6.520 & 7.3148 & 0.297 & 0.463 & - & 2.86 \\
\hline 5 & 34 & 5.08 & 5.080 & 5.7985 & 0.324 & 0.501 & 1 & 2.6 \\
\hline 6 & 35 & 6.87 & 6.713 & 7.0500 & 0.261 & 0.429 & - & 3.48 \\
\hline 7 & 36 & 5.80 & 5.800 & 6.4333 & 0.298 & 0.452 & - & 2.64 \\
\hline 8 & 37 & 5.64 & 5.640 & 5.9895 & 0.309 & 0.449 & - & 2.43 \\
\hline 9 & 38 & 8.65 & 6.628 & 5.5028 & 0.307 & 0.460 & - & 3.45 \\
\hline
\end{tabular}

Table 4. Active power rescheduling results for IEEE 39-bus system for Case-II obtained from proposed method

\begin{tabular}{c|c|c|c|c|c|c}
\hline GD unit $(i)$ & $\Delta P_{G i}{ }^{\text {hift }}$ (p.u.) & $P_{G i}{ }^{\text {ew }}$ (p.u.) & Line Outage & $P_{L O}^{\text {pre }}$ (p.u.) & GI unit $(j)$ & $P_{L O}{ }^{\text {pre }}$ (p.u.) \\
\hline 5 & 1.2514 & 3.8286 & $2-3$ & 4.4659 & 1 & 8.3603 \\
\hline & & & $14-15$ & 0.1117 & 3 & 7.2500 \\
\hline
\end{tabular}




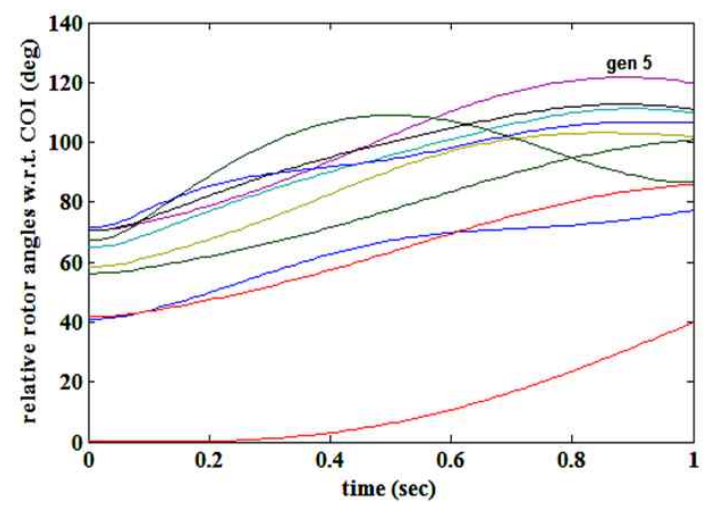

Fig. 5. Relative rotor angle trajectories of all generators of IEEE 39- bus system before rescheduling for Case II (insecure case)

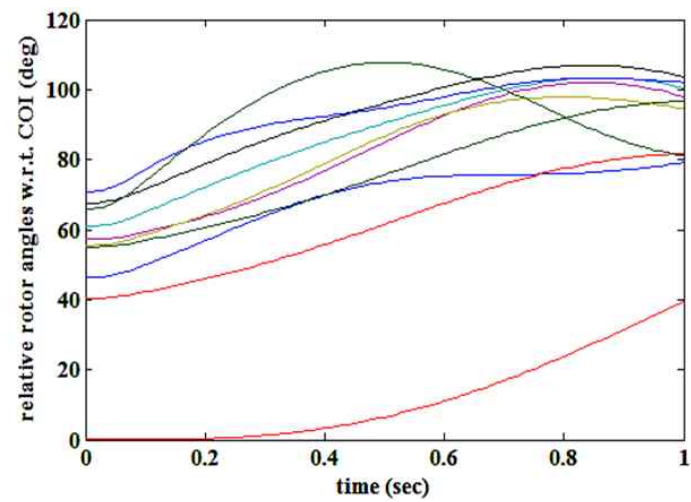

Fig. 6. Relative rotor angle trajectories of all generators of IEEE 39-bus system after proposed rescheduling for Case II (secure case)

Table 5. Results obtained from time domain simulations for IEEE 39-bus system at $105 \%$ of base case with N-1 topology variation (insecure case)

\begin{tabular}{|c|c|c|c|c|c|c|c|c|}
\hline Generator Number & Bus Number & $P_{G}^{\max }$ (p.u.) & $P_{G}^{\text {pre }}$ (p.u.) & $P_{G}^{\text {post }}$ (p.u.) & $R T I^{\text {pre }}$ & $R T I^{\text {post }}$ & Severity Rank & $\mathrm{H}(\mathrm{sec})$ \\
\hline 1 & 30 & 10.4 & 6.410 & 6.7141 & 0.147 & 0.392 & - & 4.2 \\
\hline 2 & 31 & 6.46 & 6.460 & 6.8385 & 0.306 & 0.514 & 3 & 3.03 \\
\hline 3 & 32 & 7.25 & 7.250 & 7.7259 & 0.253 & 0.476 & - & 3.58 \\
\hline 4 & 33 & 6.52 & 6.520 & 7.2550 & 0.281 & 0.505 & 4 & 2.86 \\
\hline 5 & 34 & 5.08 & 5.080 & 5.5803 & 0.305 & 0.532 & 2 & 2.6 \\
\hline 6 & 35 & 6.87 & 6.870 & 7.1672 & 0.250 & 0.479 & - & 3.48 \\
\hline 7 & 36 & 5.80 & 5.800 & 6.4001 & 0.285 & 0.496 & - & 2.64 \\
\hline 8 & 37 & 5.64 & 5.640 & 6.3973 & 0.302 & 0.504 & 5 & 2.43 \\
\hline 9 & 38 & 8.65 & 8.315 & 8.7561 & 0.331 & 0.555 & 1 & 3.45 \\
\hline
\end{tabular}

Table 6. Active power rescheduling results for IEEE 39-bus system for Case-III obtained from proposed method

\begin{tabular}{c|c|c|c|c|c|c}
\hline GD unit $(i)$ & $\Delta P_{G i}{ }^{\text {hift }}$ (p.u.) & $P_{G i}{ }^{\text {new }}($ p.u. $)$ & Line Outage & $P_{L O}{ }^{\text {pre }}($ p.u. $)$ & GI unit $(j)$ & $P_{G j}{ }^{\text {new }}($ p.u. $)$ \\
\hline 2 & 3.5500 & 2.9100 & $5-8$ & 4.016 & 1 & 10.152 \\
\hline 4 & 1.9782 & 4.5418 & & & & \\
\hline 5 & 2.3049 & 2.7751 & & & & \\
\hline 8 & 1.5044 & 4.1356 & & & & \\
\hline 9 & 4.2225 & 4.0925 & & & & \\
\hline
\end{tabular}

Table 7. Performance results of proposed rescheduling method for IEEE 39-bus system

\begin{tabular}{c|c|c|c|c|c}
\hline Case & Particulars & Total Generation (MW) & Total Load (MW) & Total power loss (MW) & System security \\
\hline \multirow{2}{*}{ Case I } & Before Rescheduling (pre-contingent) & 6207.03 & 6163 & 44.03 & Insecure \\
\cline { 2 - 7 } & After Rescheduling (post-contingent) & 6198.26 & 6163 & 35.26 & Secure \\
\hline \multirow{2}{*}{ Case II } & Before Rescheduling (pre-contingent) & 6348.28 & 6303.8 & 44.48 & Insecure \\
\cline { 2 - 7 } & After Rescheduling (post-contingent) & 6372.88 & 6303.8 & 69.08 & Secure \\
\hline \multirow{2}{*}{ Case III } & Before Rescheduling (pre-contingent) & 6687.89 & 6640.1 & 47.79 & Insecure \\
\cline { 2 - 7 } & After Rescheduling (post-contingent) & 6690.58 & 6640.1 & 50.48 & Secure \\
\hline
\end{tabular}

\section{Case III: N-1 Topology Variation; 105\% of Base Case}

This case study is shown for $\mathrm{N}-1$ topology variation with randomly changing load at $105 \%$ of base case. Single line outage of line 5-8 is considered with three-phase fault at bus-26. As shown in Table 5, the results obtained from TDS indicate that the post-contingent value $R T I^{\text {post }}$ for the generator no. $9,5,24$, and 8 exceeds $\varepsilon$ (in the order of their severity ranking) and hence they lose synchronism with rest of the system. Table 6 show the proposed active power rescheduling among $C M S$ and $N M s$ to bring the current insecure operating state to a secure state. It is found that the GI group consists of only one unit, which is generator no. 1 having lowest value of $R T I^{\text {post }}$.

The generation decrease is required for all five critical machines while generation increase for only one noncritical generating unit is sufficient to bring the system back to secure state. As seen from Fig. 7, relative rotor angle of generator no. 2, 4, 5, 8 and 9 increases beyond the threshold value, $\varepsilon$ and goes out of step before rescheduling. These machines lose synchronism with rest of the generators making system transiently insecure. As shown 


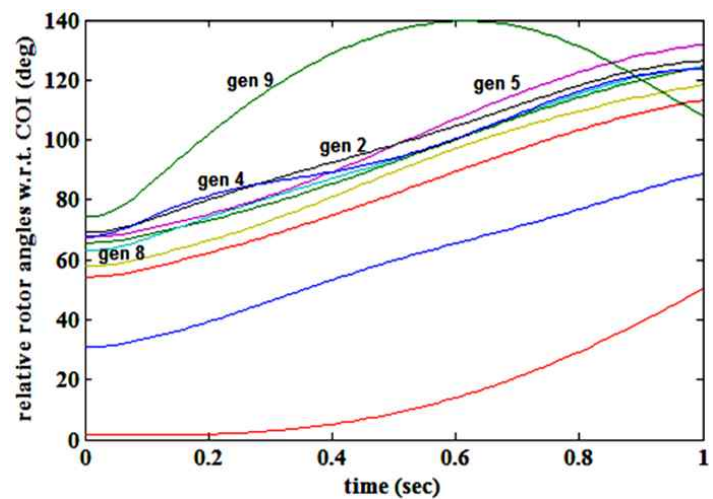

Fig. 7. Relative rotor angle trajectories of all generators of IEEE 39-bus system before rescheduling for Case III (insecure case)

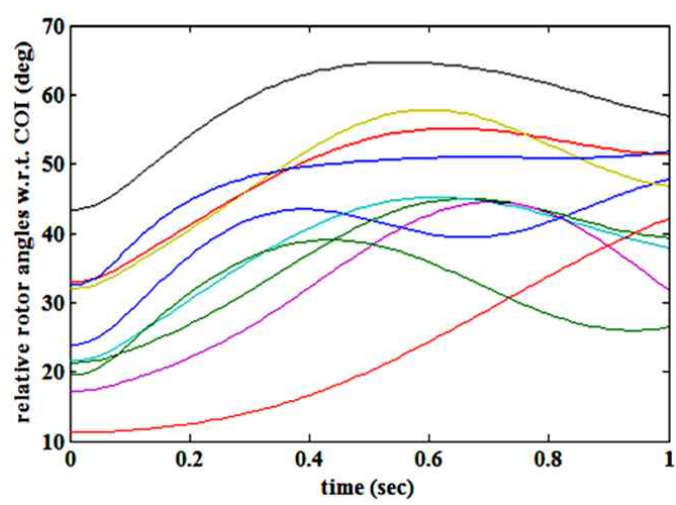

Fig. 8. Relative rotor angle trajectories of all generators of IEEE 39-bus system after proposed rescheduling for Case III (secure case)

in Fig. 8, after proposed rescheduling, the relative rotor angle of all the generating units is below $\varepsilon$ and the system becomes secure.

The performance analysis and the effectiveness of proposed method is validated for all the cases as shown in Table 7 indicating the transient security status and total system real power losses. With the pre-contingent schedules if there is 3-phase fault on the system, it becomes transiently insecure.

\section{Conclusions}

In modern large and interconnected power systems, system security is paramount. Under normal operating conditions, power systems operate in economic mode with minimum possible operating cost. However, if any operating state is found to be insecure, then system security takes over system economy and the preventive action may be taken to bring the state in secure operating state even if it causes slight loss in economy. In this paper, generator rescheduling based on rotor trajectory index $(R T I)$ is proposed. The application results are investigated on IEEE 39-bus, 10-generator system under different operating conditions. The proposed method of generators rescheduling is capable of converting insecure operating into secure operating state by modifying the generators' dispatch. The information about ranking obtained from $R T I$ of each generating unit is important for selecting the units for generation decrease. The proposed method can be suitably employed for preventive control of power systems even under multiple contingency conditions.

\section{References}

[1] Ruiz-Vega, D., Pavella, M., "A Comprehensive Approach to Transient Stability Control: Part I: Near Optimal Preventive Control, Part II: Open Loop Emergency Control", IEEE Transactions on Power Systems, Vol.18, pp.1446-1460, 2003.

[2] IEEE/CIGRE Joint Task Force on Stability Terms and Definitions, "Definition and classification of power system stability", IEEE Transactions on Power Systems, Vol. 19 (2), pp.1387-1401, 2004.

[3] P. Kundur, Power System Stability and Control, McGraw Hill, 1994.

[4] K. R. Padiyar, Power System Dynamics: Stability and Control, New York: Wiley, 1996.

[5] Wehenkel L, Pavella M., "Preventive vs. Emergency Control of Power Systems", IEEE/Power Energy Society Power Systems Conference \& Exposition, pp. 1-6, 2004.

[6] D. H. Kuo, A. Bose: 'A Generation Rescheduling Method to Increase the Dynamic Security of Power Systems', IEEE Transactions on Power Systems, Vol. 10 (1), pp. 68-76, 1995.

[7] W. Li, A. Bose, "A Coherency Based Rescheduling Method for Dynamic Security", IEEE Transactions on Power Systems, Vol.13 (3), pp. 810-815, 1998.

[8] Kusum Verma, K. R. Niazi, "A Coherency Based Generator Rescheduling for Preventive Control of Transient Stability in Power Systems", Electrical Power and Energy Systems, Vol. 45 (1), pp. 10-18, 2013.

[9] C.F. Kucuktezcan, V.M.I. Genc, "A New Dynamic Security Enhancement Method via Genetic Algorithms Integrated With Neural Network Based Tools", Electric Power Systems Research, Vol. 83, pp. 1-8, 2012.

[10] M. K. Maharana, K.S. Swarup, "Graph Theoretic Approach for Preventive Control of Power Systems", Electrical Power and Energy Systems, vol. 32 (4), pp. 254-261, 2010.

[11] Y. J. Lin., "Prevention of Transient Instability Employing Rules Based on Back Propagation Based ANN for Series Compensation”, Electrical Power and Energy Systems, Vol. 3, pp. 1776-1783, 2011.

[12] Fang DZ, Yang X, Sun J, Yuan S, Zhang Y., “An 
Optimal Generation Rescheduling Approach for Transient Stability Enhancement", IEEE Transactions on Power Systems, Vol. 22(1), pp. 386-394, 2007.

[13] M. Kheradmandi, M. Ehsan, R.Feuillet, N. HadjSaied, "Rescheduling of Power Systems Constrained with Transient Stability Limits in Restructured Power Systems", Electric Power Systems Research, Vol. 81, pp. 1-9, 2011.

[14] D.Z. Fang, W. Sun, Z.Y. Xue, "Optimal Generation Rescheduling With Sensitivity-Based Transient Stability Constraints", IET Generation Transmission Distribution, Vol.4 (9), pp.1044-1051, 2010.

[15] Y. Yuan, L. Zuo, X. Yuan, J. Kubokawa, H. Sasaki, “A Novel Method for Power System Transient Stability Preventive Control", IEEE/PES Trans-mission and Distribution Conference \& Exhibition, China, pp.1-6, 2005

[16] V. Miranda, J.N. Fidalgo, J.A.P. Lopes, L.B. Almeida, "Real Time Preventive Actions for Transient Stability Enhancement with a Hybrid Neural Network-Optimization Approach", IEEE Transactions on Power Systems, Vol. 10(2), pp. 1029-1035, 1995.

[17] Tony B. Nguyen and M. A. Pai, "Dynamic SecurityConstrained Rescheduling of Power Systems using Trajectory Sensitivities", IEEE Transactions on Power Systems, Vol. 18(2), pp. 848-854, 2003.

[18] Li YH, Yuan WP, Chan KW, Liu MB., "Coordinated Preventive Control of Transient Stability with MultiContingency in Power Systems using Trajectory Sensitivities", Electrical Power and Energy Systems, Vol. 33(1), pp. 147-53, 2011.

[19] D. Gan, Z. Qu, H. Cai, X. Wang, "Methodology and Computer Package for Generation Rescheduling", IEE Proceedings Generation, Transmission \& Distribution, Vol. 144(3), pp. 301-307, 1997.

[20] M. Djukanovic, D.J. Sobajic, Y.H. Pao, "Neural Net Based Determination of Generator-Shedding Requirements in Electric Power Systems", IEE ProceedingsC, Vol.139(5), pp.427-436, 1992.

[21] I. Genc, R. Diao, V. Vittal, S. Kolluri, and S. Mandal, "Decision Tree-Based Preventive and Corrective Control Applications for Dynamic Security Enhancement in Power Systems", IEEE Transactions on Power Systems, Vol. 25 (3), pp. 1611-1619, 2010.

[22] E.M. Voumvoulakis, N.D. Hatziargyriou, "A Particle Swarm Optimization Method for Power System Dynamic Security Control", IEEE Transactions on Power Systems, Vol. 25(2), pp.1032-1041, 2010.

[23] R. Minano, T. V. Custem, F. Milano, A. J. Conejo, "Securing Transient Stability Using Time-Domain Simulations Within An Optimal Power Flow", IEEE Transactions on Power Systems Vol. 25(1), pp. 243253, 2010

[24] Power System Test Cases Available at http://www.ee.washington.edu/pstca/

[25] The Math Works, Inc., MATLAB programming, 2008.
[26] Graham Rogers, Power System Toolbox (PST), User's Guide, 2008

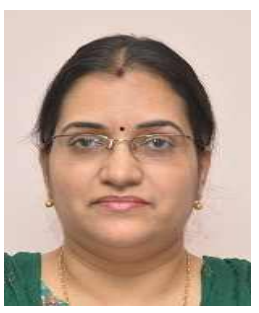

Kusum Verma She received her B.E. in Electrical Engineering from Rajasthan University, Jaipur, India in 1997, M. Tech. in Electrical Engineering (Power Systems) in 2004 and Ph.D. in Electrical Engineering in 2013 from Malaviya National Institute of Technology, Jaipur, Rajasthan, India. Presently she is working as Assistant Professor in Department of Electrical Engineering at Malaviya National Institute of Techno logy, Jaipur, India. Her research interests include power system operation and control, power system security assessment, application of artificial intelligence to power systems.

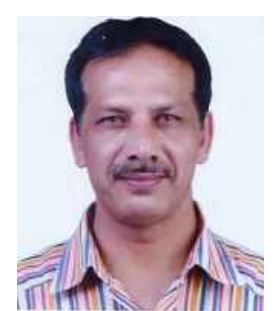

K. R. Niazi He received his B.E. in electrical engineering from Rajasthan University, Jaipur in 1987, M.E. in Electrical Engineering (Control Systems) from JNV University, Jodhpur in 1997, and Ph.D. in Electrical Engineering from Rajasthan University, Jaipur in 2003. He has been working as Professor in Electrical Engineering at Malaviya National Institute of Technology, Jaipur, India. Presently he is Professor in Department of Electrical Engineering at Taibah University, Madinah, KSA. His areas of research interest are power system optimization, security analysis, distribution system optimization and application of artificial neural network to power systems. 